\title{
Supporting information on:
}

\section{Surface Reconstruction Under the Exposure of}

\section{Electric Fields Enhances the Reactivity of Donor-}

\section{doped $\mathrm{SrTiO}_{3}$}

Buğra Kayaalp ${ }^{1}$, Kurt Klauke ${ }^{1}$, Mattia Biesuz $^{2}$, Alessandro Iannaci $^{3}$, Vincenzo M. Sglavo², Massimiliano D'Arienzo ${ }^{4}$, Heshmat Noei ${ }^{5}$, Siwon Lee ${ }^{6}$, WooChul Jung ${ }^{6}$ and Simone Mascotto ${ }^{*}$

${ }^{1}$ Institut für Anorganische und Angewandte Chemie, Universität Hamburg, Martin-Luther-KingPlatz 6, 20146 Hamburg, Germany

2Dipartimento di Ingegneria Industriale, Università degli Studi di Trento, Via Sommarive 9, 38123 Trento, Italy

${ }^{3}$ INSTM, Trento Research Unit, Via G. Giusti 9, 501211 Florence, Italy

${ }^{4}$ Dipartimento di Scienze dei Materiali, Università di Milano-Bicocca, Via R. Cozzi, 55, 20125 Milano, Italy

${ }^{5}$ Deutsches Elektronen-Synchrotron DESY, Notkestr. 85, 22607 Hamburg, Germany

${ }^{6}$ Department of Materials Science and Engineering, Korea Advanced Institute of Science and Technology, 291 Daehak-ro, Yuseong-gu, Daejeon 34141, Republic of Korea

Corresponding Author

* simone.mascotto@chemie.uni-hamburg.de 


\section{Characterization of as-prepared $\mathrm{La}_{0.1} \mathrm{Sr}_{0.9} \mathrm{TiO}_{3}$ nanoparticles}

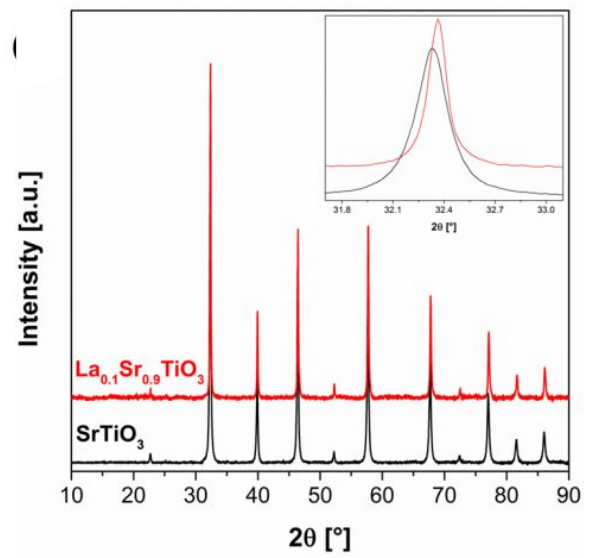

Figure S1: X-ray diffraction patterns of as-prepared (AP) $\mathrm{SrTiO}_{3}$ and $\mathrm{La}_{0.1} \mathrm{Sr}_{0.9} \mathrm{TiO}_{3}$ (LSTO) nanoparticles. The shift of [110] reflection due to doping is presented in the inset.

The shift of the (110) reflection to higher $2 \theta$ with respect to pure $\mathrm{SrTiO}_{3}$ in Fig. S1c suggests the substitution of $\mathrm{Sr}^{2+}(r=1.44 \AA)$ with the smaller $\mathrm{La}^{3+}$ ion $(r=1.36 \AA)$ in the perovskite lattice ${ }^{1}$, and therefore the successful doping of the parent $\mathrm{SrTiO}_{3}$ structure.

\section{Consolidation process}

(a)

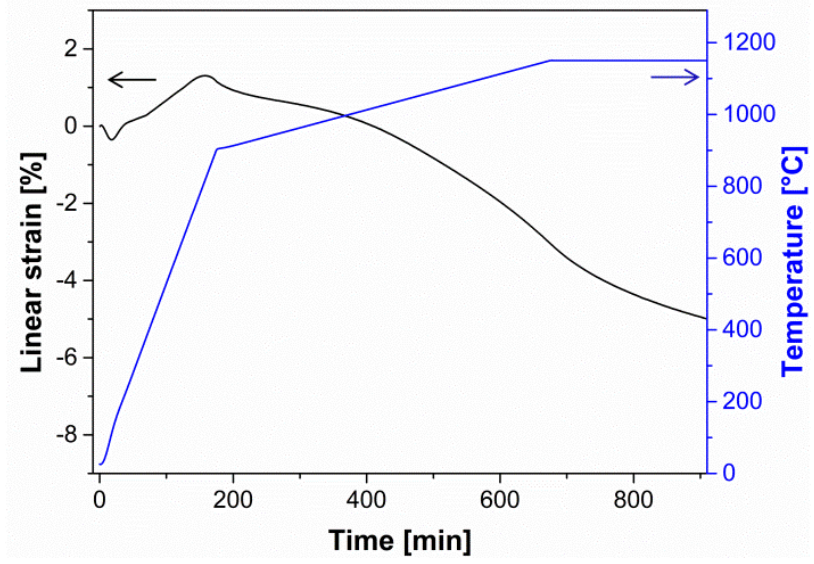

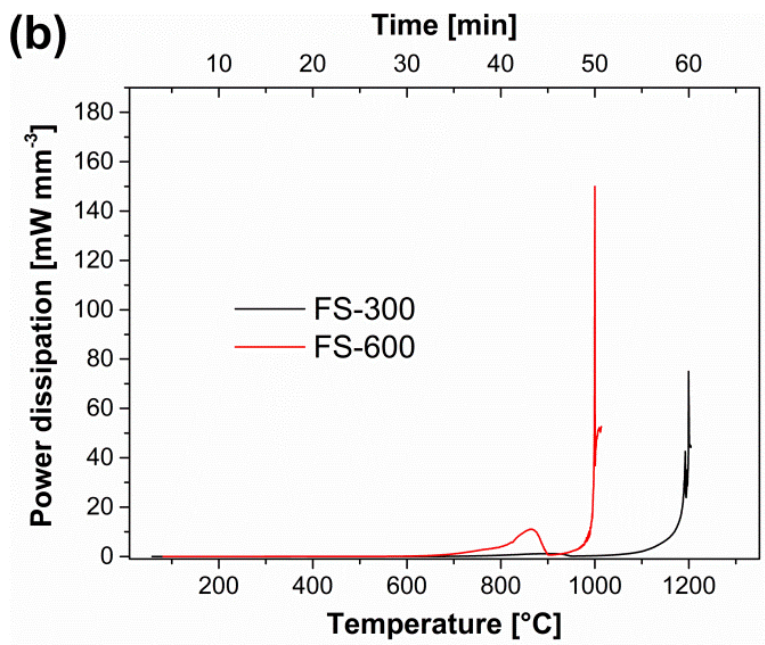

Figure S2: Percentage shrinkage of LSTO by conventional sintering (a) and power dissipation plots for flash sintering of LSTO (b). 
The initial expansion and shrinkage observed on Fig S2a corresponds to the transitions i.e. removal of the binder material. The consolidation during a field-assisted process is strongly related to the electrical power dissipation within the ceramic component ${ }^{2,3}$. The power peaks reached in this work were 75 and $150 \mathrm{~mW} \mathrm{~mm}^{-3}$ for the sample treated with 300 (FS-300) and $600\left(\right.$ FS-600) V cm${ }^{-1}$, respectively (Fig. S2b). Such values are about one order of magnitude lower than the typical power peaks reached during standard flash sintering, which enable total and effective densification ${ }^{2,4}$.

\section{Surface area investigations}

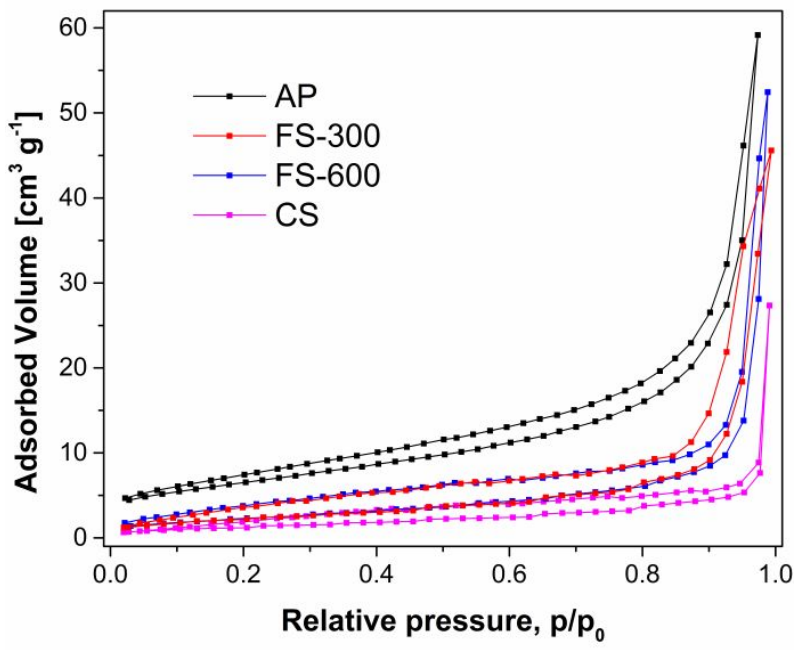

Figure S3: $\mathrm{N}_{2}$ physisorption isotherms of investigated LSTO systems. 


\section{Crystallographic characterization of FS-600}

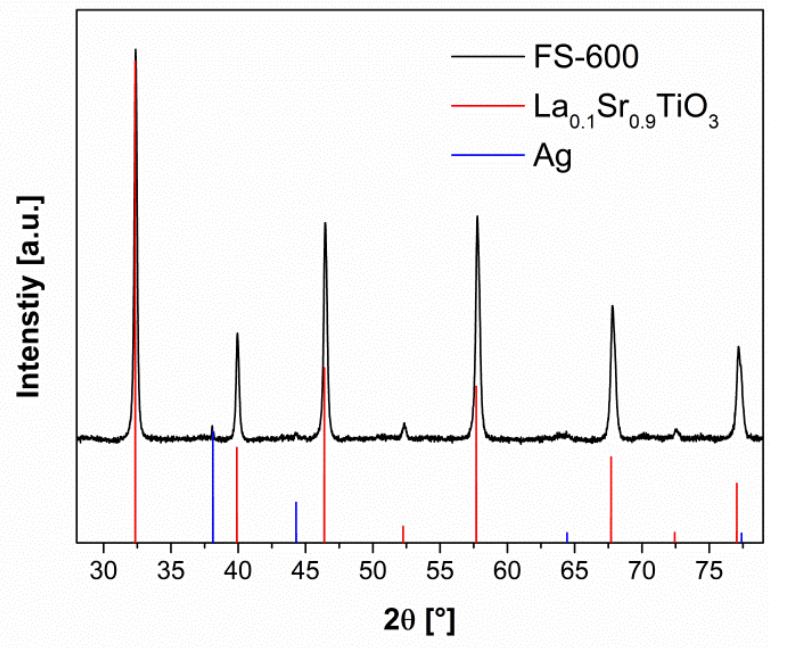

Figure S4: X-ray diffractograms of FS-600 along with reference diffractograms of $\mathrm{La}_{0.1} \mathrm{Sr}_{0.9} \mathrm{TiO}_{3}(\mathrm{ICSD}$ Code: 065091) and Ag (ICSD Code: 064706) obtained from ICSD database.

\section{Morphology and composition investigations}
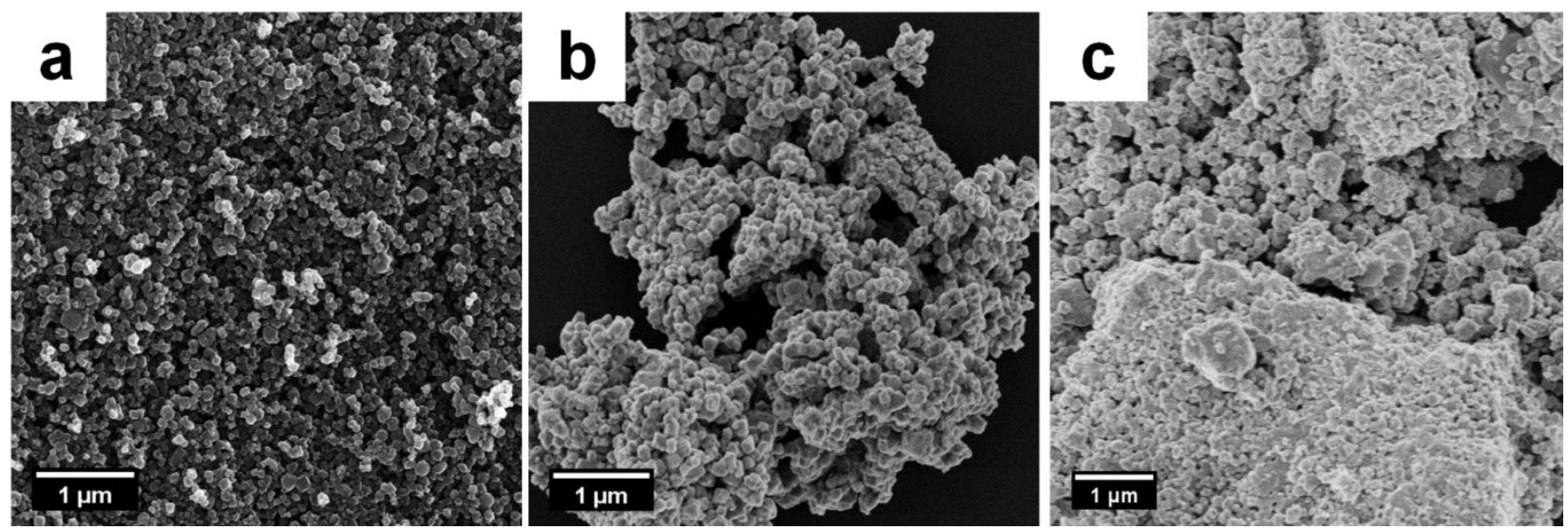

Figure S5: SEM micrographs of investigated LSTO systems (a) AP (b) FS-600(c) CS. 

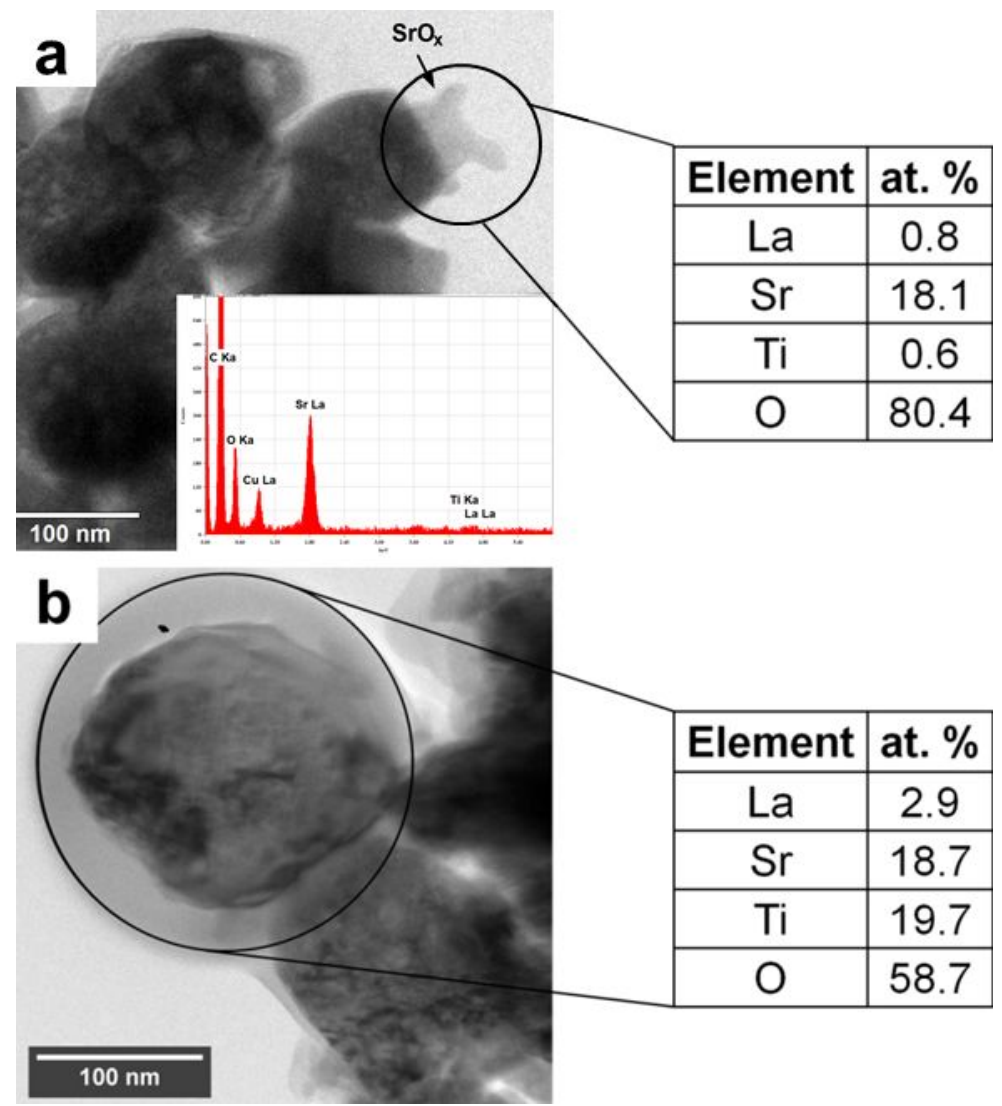

Figure S6: Atomic compositions acquired from TEM/EDX over the circular area of investigated LSTO samples (a) FS-600 (b) CS.

Formation of higher contrast $\mathrm{SrO}_{\mathrm{x}}$ rich sites as a result of flash sintering was confirmed by EDX spectroscopy (Fig. S6a), whereas no exclusive $\mathrm{SrO}_{\mathrm{x}}$ sites were detected for conventionally sintered sample (Fig. S6b). 

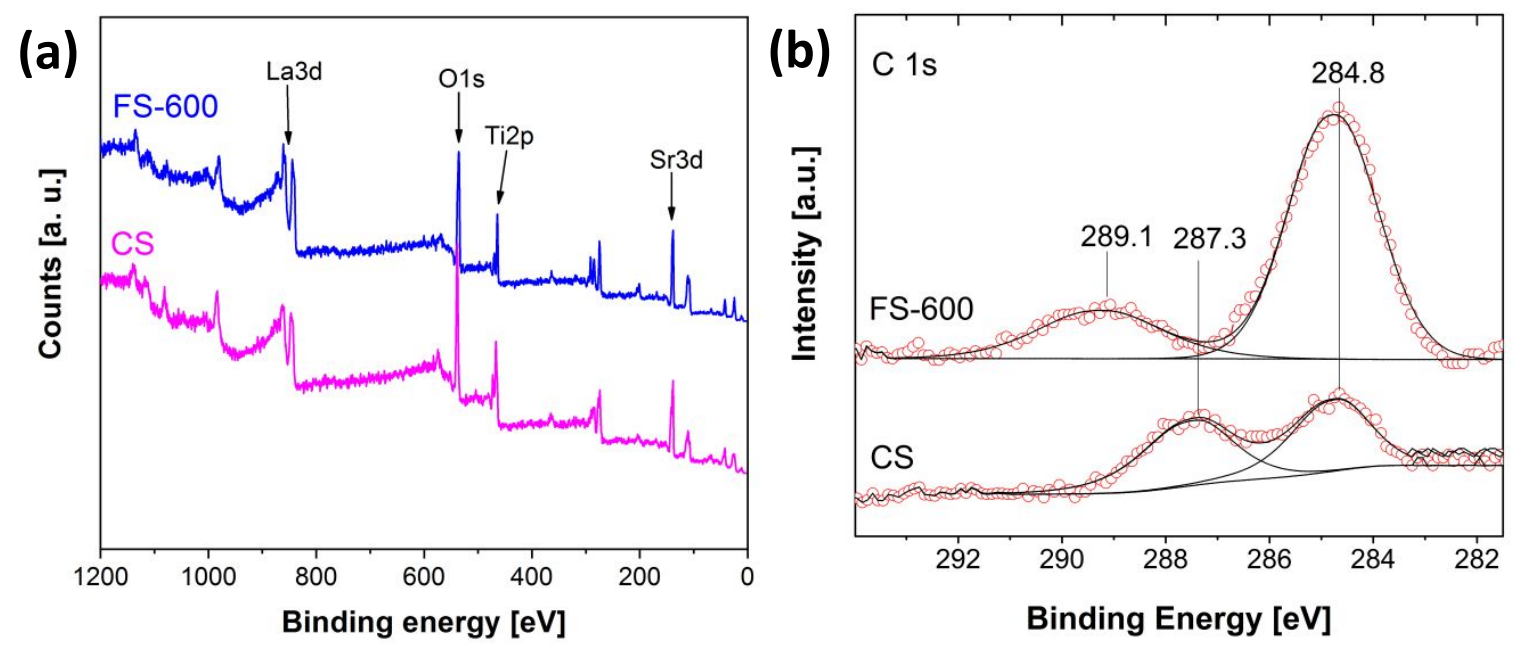

Figure S7: XPS surveys of the CS and FS-600 samples identifying the characteristic elements of the ceramics (a); C 1s region of the CS and FS-600 sample showing the surface carbon contaminations (b).

Concerning the $\mathrm{C} 1 \mathrm{~s}$ region of the XPS, the three signals observed are ascribed to the adventitious hydrocarbon $\mathrm{C}-\mathrm{C} / \mathrm{C}-\mathrm{H}$ bonds $(\mathrm{BE}=284.8 \mathrm{eV})$, and to carbooxides containing $\mathrm{C}-\mathrm{O}-\mathrm{C}(\mathrm{BE}=287$. $3 \mathrm{eV})$ and $\mathrm{O}-\mathrm{C}=\mathrm{O}(\mathrm{BE}=289.1 \mathrm{eV})$ bonds ${ }^{5,6}$. The presence of large concentration of carbonate species on the FS-600 surface is demonstrated by the signal at $298.1 \mathrm{eV}^{6}$ and is likely ascribed to $\mathrm{SrCO}_{3}$ given the large $\mathrm{Sr}^{2+}$ segregation observed in this sample

\section{ESR spectroscopy}

Table S1: $g$ tensor values of the paramagnetic defects detected over FS-600 sample, determined by simulation of the ESR features. The relative contributions of the $\mathrm{O}^{-}$and $\mathrm{Ti}^{3+}$ species (calculated as $\%$ of the total signal intensity) are also reported.

\begin{tabular}{|c|c|c|}
\hline Species & $g$-values & Contribution [\%] \\
\hline$O^{-}{ }_{[I]}$ & $g_{\perp}=2.0550, g_{\|}=1.9950$ & 45 \\
\hline$O^{-}{ }_{[I I]}$ & $g_{\perp}=2.0030, g_{\|}=1.9990$ & 22 \\
\hline$O^{-}{ }_{[I I]}$ & $g_{\perp}=2.0190, g_{\|}=1.9970$ & 12 \\
\hline$O^{-}{ }_{[I V]}$ & $g_{\perp}=2.0350, g_{\|}=2.0030$ & 6 \\
\hline$T i^{3+}$ & $g=1.979$ & 15 \\
\hline
\end{tabular}




\section{Catalytic investigations}

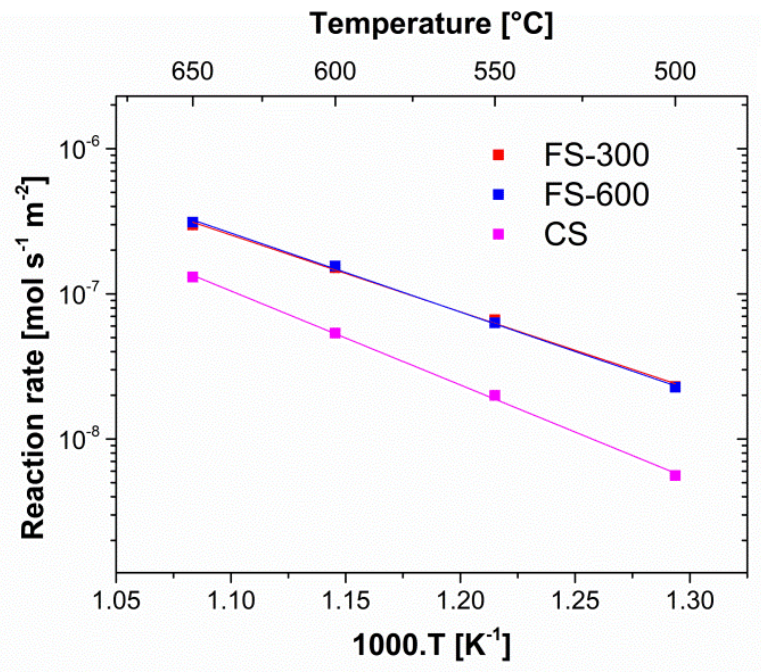

Figure S8: Arrhenius-type plots obtained from catalytic methane oxidation tests over the consolidated materials. Reaction rates are normalized by specific surface area of each sample and the calculated activation energies are reported on Table 1.

\section{References:}

(1) Bamberger, C. E. Homogeneity Ranges of Phases Sr4-xLn2x/3Ti4O12 ( $\operatorname{Ln}=\mathrm{Sm}$ to $\mathrm{Lu})$ and Sr1-yEuy\|lTiO3. J. Am. Ceram. Soc. 2000, 26, 1996-1998.

(2) Biesuz, M.; Sglavo, V. M. Flash Sintering of Alumina: Effect of Different Operating Conditions on Densification. J. Eur. Ceram. Soc. 2016, 36, 2535-2542.

(3) Baraki, R.; Schwarz, S.; Guillon, O. Effect of Electrical Field/Current on Sintering of Fully Stabilized Zirconia. J. Am. Ceram. Soc. 2012, 95, 75-78.

(4) Caliman, L. B.; Bichaud, E.; Soudant, P.; Gouvea, D.; Steil, M. C. A Simple Flash Sintering Setup under Applied Mechanical Stress and Controlled Atmosphere. MethodsX 2015, 2, 392-398.

(5) Greczynski, G.; Hultman, L. C 1s Peak of Adventitious Carbon Aligns to the Vacuum Level: Direct Consequences for Material's Bonding Assignment by Photoelectron Spectroscopy. Chemphyschem 2017, 18, 1507-1512. 
(6) Nagarkar, P. V.; Searson, P. C.; Gealy, F. D. Effect of Surface Treatment on SrTiO3: An x-Ray Photoelectron Spectroscopic Study. J. Appl. Phys. 1991, 69, 459-462. 